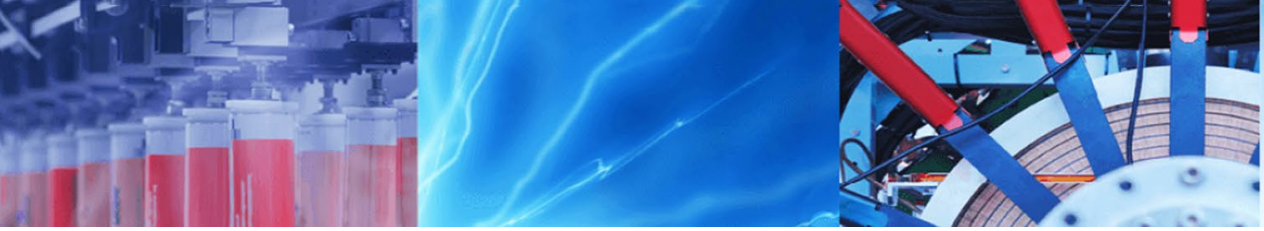

Research Article

\title{
Tetragonal tungsten bronze/barium hexaferrite room-temperature multiferroic composite ceramics
}

\author{
Thameur Hajlaoui $^{1}$ (1) $\cdot$ Mohsen Elain Hajlaoui ${ }^{2} \cdot$ Michaël Josse $^{3} \cdot$ Essebti Dhahri $^{2} \cdot$ Alain Pignolet $^{1}$
}

Received: 3 August 2020 / Accepted: 13 October 2020 / Published online: 22 October 2020

(c) Springer Nature Switzerland AG 2020

\begin{abstract}
The spontaneously formed multiferroic composite based on tetragonal tungsten bronze structure was successfully synthesized in the form of ceramics by the solid-state reaction. The crystallization of the ceramics in the tetragonal tungsten bronze structure $\mathrm{Ba}_{2} \mathrm{SmFeNb}_{4} \mathrm{O}_{15}$ and the presence of a secondary magnetic phase of barium hexaferrite $\mathrm{BaFe}_{12} \mathrm{O}_{19}$ were established by structural investigation. The hysteresis behavior describing the polarization as a function of an applied electric field and the high ferroelectric Curie temperature $\approx 417 \mathrm{~K}$ evidenced the room-temperature ferroelectric properties of the synthesized material. While the piezoelectric coefficients were determined to be around $1.3 \mathrm{pm} / \mathrm{V}$, the microscopic polar domains of the composite ceramics were established by microelectromechanical study. The hysteresis loop of the magnetization versus magnetic field and the high magnetic transition temperature $\approx 590 \mathrm{~K}$ evidenced the ferromagnetic properties of the secondary phase and its presence at room temperature. Moreover, the spatial distribution of the magnetic domains was determined microscopically. In this study, not only the multiferroic properties of the $\mathrm{Ba}_{2} \mathrm{SmFeNb}_{4} \mathrm{O}_{15} / \mathrm{BaFe}_{12} \mathrm{O}_{19}$ composites have been studied and presented, but the distribution of the polar and magnetic properties was locally investigated as well.
\end{abstract}

Keywords Composite · Room-temperature multiferroic · Electromechanical properties · Polar domains · Magnetic domains

\section{Introduction}

Recently, multiferroic/multifunctional materials have attracted the interest of scientists due to their rich properties and their potential use in a wide range of advanced applications [1-5]. Especially, materials with double ferroic ordering, in particular exhibiting ferroelectric and ferromagnetic properties [6-8], are widely investigated because of their potential use in various applications [9, 10], including new generations of non-volatile integrated memories [11-13].

In order to overcome the issue of the scarcity of singlephase multiferroic materials, especially at the ambient temperature and above, several strategies have been adopted in order to develop new multiferroic materials at room temperature [14-17]. One of the most efficient ways is to synthesize composites materials formed by ferroelectric and ferromagnetic phases, which allows the use of a large variety of ferroelectric/piezoelectric and magnetic material couples, having both good ferroelectric and good ferromagnetic properties [5, 18-21]. An important issue with composites is the chemical compatibility of the targeted phases, and the formation of secondary phases (or of interphases) can be complex and challenging [22, 23].

Tetragonal tungsten bronzes (TTB) are a class of materials having a crystal structure consisting of a framework of

$\triangle$ Thameur Hajlaoui, thameur.hajlaoui@emt.inrs.ca | ${ }^{1}$ Institut National de la Recherche Scientifique - Énergie Matériaux et Télécommunications, 1650 Boulevard Lionel-Boulet, Varennes, QC J3X 1S2, Canada. ${ }^{2}$ Laboratory of Applied Physics, Faculté des Sciences de Sfax, Université de Sfax, B.P. 1171, 3000 Sfax, Tunisia. ${ }^{3}$ Univ. Bordeaux, ICMCB, UPR 9048, 33600 Pessac, France. 
corner-sharing octahedra connected in such a way that different channels with different shapes (triangular, square and pentagonal channels) are formed. These channels are all oriented along the short crystallographic c-axis and can be filled, partially filled or empty. Such a crystal structure with a large number of cationic and anionic sites allows for the synthesis of large variety of composites and dopings, resulting in a fine control and a large enhancement of the functional properties of the synthesized material [24-27]. Crystalizing in this particular structure, the ferroelectric tetragonal tungsten bronze $\mathrm{Ba}_{2} \mathrm{LnFeNb}_{4} \mathrm{O}_{15}$ (TTB-Ln), with $\mathrm{Ln}$ being a lanthanide, has been shown to exhibit a particular behavior, namely the in situ generation of ferromagnetic barium hexaferrite $\left(\mathrm{BaFe}_{12} \mathrm{O}_{19}\right.$ : $\left.\mathrm{BaFO}\right)$, during the proper sintering of the ferroelectric matrix $[24,28,29]$. This process therefore produces a spontaneously forming multiferroic ceramic composite and overcomes any chemical compatibility issue that are common with conventional composites. The magnetoelectric coupling that represents the correlation between the ferroelectric and the magnetic properties in these materials has been recently studied by Cheng Li et al. [30]. The authors showed that the magnetoelectric coupling coefficient of these ceramics is around $0.27 \mathrm{mV} / \mathrm{Oe}$ at an applied magnetic field of around $300 \mathrm{Oe}$.

For complete understanding of the multiferroic properties and their microscopic distribution, this contribution reports a complementary investigation of the ferroelectric and ferromagnetic properties of $\mathrm{Ba}_{2} \mathrm{SmFeNb}_{4} \mathrm{O}_{15}$ (TTB$\mathrm{Sm}) / \mathrm{BaFe}_{12} \mathrm{O}_{19}(\mathrm{BaFO})$ composite ceramics synthesized by solid-state reaction [24]. By studying the dielectric coefficients and the magnetization versus the temperature, we determined the ferroelectric and the ferromagnetic transition temperatures, which confirms the room-temperature multiferroic nature of the composite. In addition, we used microelectromechanical investigations to study the polar domains at the ceramics surface and determine their piezoelectric coefficients. We also studied the magnetic response at the microscopic level, and we determined the spatial distribution of the magnetic secondary phase.

\section{Materials and methods}

Tetragonal tungsten bronze multiferroic composite ceramics were prepared by solid-state reaction method. The precursors $\mathrm{BaCO}_{3}, \mathrm{Fe}_{2} \mathrm{O}_{3}, \mathrm{Nb}_{2} \mathrm{O}_{5}$ and $\mathrm{Sm}_{2} \mathrm{O}_{3}$ (having a purity higher than 99\%) were mixed by mechanical grinding and pressed under uniaxial pressure of $4 \mathrm{ton} / \mathrm{cm}^{2}$ into cylindrical pellets with a diameter of $8 \mathrm{~mm}$ and a thickness around $1.2 \mathrm{~mm}$ before being sintered at $1300^{\circ} \mathrm{C}$ during $4 \mathrm{~h}$ in air. The ceramics crystallization and phase identification were characterized at room temperature by $\mathrm{X}$-ray diffraction collected using a 4-circle X-ray diffractometer (PANalytical
X-Pert PRO MRD). In order to prepare the samples for ferroelectric and dielectric measurements, we deposited, on each side of the pellet, a 200-nm-thick layer of gold by using thermal evaporation under vacuum giving diskshaped electrodes of $6 \mathrm{~mm}$ dia each. Dielectric characteristics were performed with Wayne-Kerr 6425 component analyzer under dry helium at frequencies ranging from $10^{3}$ to $10^{6} \mathrm{~Hz}$. The electric field dependence of the polarization was measured using an analogical Sawyer-Tower circuit with compensation of stray capacitances and resistances. The microstructure, the local electromechanical properties and the microscopic magnetic response were characterized by an atomic force microscope (AFM) using a DI-enviroscope AFM (Bruker, Santa Barbara, CA). To image and manipulate the ferroelectric polarization of the samples at the nanoscale, we used piezoresponse force microscopy (PFM) and we applied a voltage of $3 \mathrm{~V}$ at $20 \mathrm{kHz}$ between the conductive tip and a silver past bottom electrode. The surface-induced piezoelectric vibrations were detected using a lock-in amplifier from Signal Recovery (model 7265, Wokingham, UK). Magnetic force microscopy (MFM) was performed to investigate the local magnetic response of the samples. The microscopic measurements were carried out with conductive/magnetic tips, having a $\mathrm{Co} / \mathrm{Cr}$ coating (MESP from Bruker, Santa Barbara, $(\mathrm{A})$, thus suitable for both electric and magnetic measurements. To avoid any possible artifact due to the sample topography in the magnetic response, the MFM measurements were performed in the so-called lift mode, where two passes are performed while scanning each line of the sample surface; during the first pass (scanning in tapping mode), the tip height is used to determine and record the surface topography of the sample. A second pass on the same line is then performed at a constant but larger distance from the sample surface $(\approx 100 \mathrm{~nm})$-taking into account the topography recorded during the first passin order to solely measure the magnetic response. (The magnetic interactions were detected via the resonance frequency shift.) At the macroscopic level, the variation of the magnetization as a function of temperature and as a function of an applied magnetic field was studied using an EV9 Vibrating Sample Magnetometer (VSM) produced by Microsense (Lowell, MA, USA).

\section{Results and discussion}

Figure 1 shows the $\mathrm{X}$-ray diffractogram of the composite ceramics. The diffraction peaks shown with the Miller indices are attributed to the principal phase TTB-Sm according to the reference file JCPDS 00-059-0425, confirming that the principal phase crystallizes in the tetragonal system with the tungsten bronze structure belonging to with the 
Fig. 1 X-ray diffractogram of the synthesized TTB-Sm/BaFO ceramic composite: in addition to the diffraction peaks of the TTB-Sm (shown with their Miller indices), the peaks of the secondary phases $\mathrm{BaFe}_{12} \mathrm{O}_{19}$ and $\mathrm{SmNbO}_{4}$ are shown with the symbols $(\cdot)$ and (\#), respectively. The inset displays a unit cell of TTB-Sm, indicating the different channels with pentagonal (filled with $\mathrm{Ba}^{2+}$ ), square (filled with $\mathrm{Sm}^{2+}$ ) and triangular (empty) shapes and the positions of the different ions

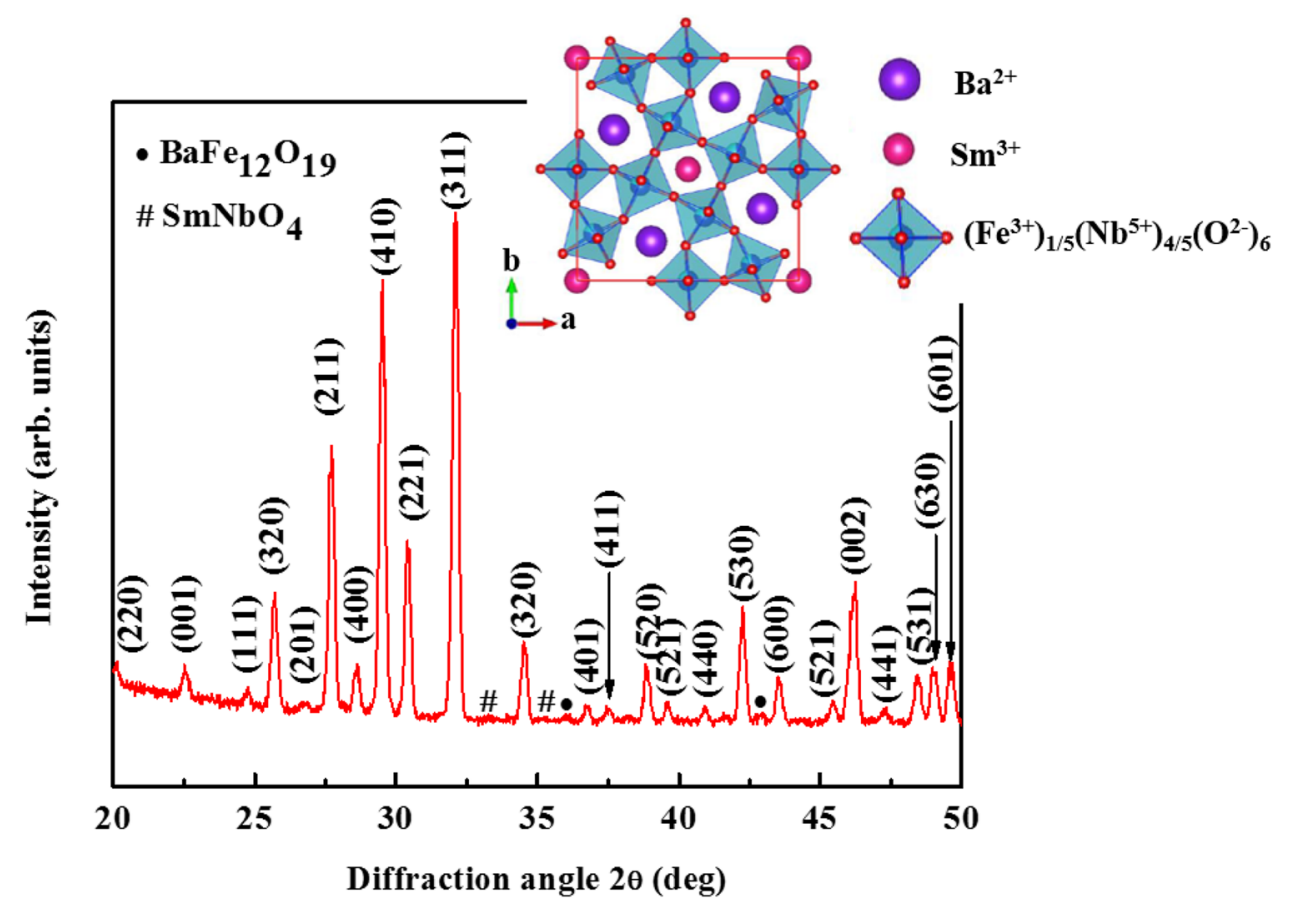

space group Pba2. The detailed study of the structural properties of the synthesized ceramics has been previously reported in reference [24]. In particular, the lattice parameters of the tetragonal structure were determined to be $a \approx 12.458 \AA$ and $b \approx 3.928 \AA$ [24]. The crystallite size is determined to be around $35 \mathrm{~nm}$ using Scherrer's formula [31], which is in accordance with [32]. A sketch of the TTB-Sm crystal structure seen along the crystallographic C-axis is drawn using the software VESTA, and the crystallographic data provided in $[24,33]$ are shown in the inset of Fig. 1. In this structure, the barium ions occupy the pentagonal sites, the samarium ions are contained in the square sites, while the triangular sites are kept empty. The $\mathrm{Fe}^{3+}$ and $\mathrm{Nb}^{5+}$ metal ions are statistically distributed within oxygen octahedral, which are responsible for the appearance of the ferroelectric polarization [24, 29]. Moreover, $\mathrm{SmNbO}_{4}$ phase (\#) is always detected, due to the limited structural distortions of the TTB phase, which results only in a partial accommodation of the samarium ions $[24,29]$. The remaining ions interact with a small amount of niobium and oxygen to form the fergusonite phase. In order to maintain electrical neutrality, iron ions can only be partially incorporated in the TTB framework and the excess portion of iron oxide reacts with a small amount of barium oxide in order to form the BaFO phase (•) $[24,28,34]$. The fergusonite phase has no ferroic properties in the investigated temperature range, but its presence is required to trigger the formation of the ferromagnetic hexaferrite phase within the TTB matrix [24]. In addition, the formation of the magnetic BaFO phase is very desirable because of the large use of this phase in several applications, such magnetic recording and microwave applications [35-37].

To investigate the ferroelectric properties in the prepared ceramics, we studied the polarization hysteresis loops obtain by applying an electric field of various amplitude varying form $12 \mathrm{kV}$ to $26 \mathrm{kV} / \mathrm{cm}$. The dependence on the electric field of the global polarization (P-E) and the current (I-E) across a pellet of ceramic sandwiched between two gold electrodes is shown in Fig. 2a, b, respectively. The hysteresis behavior that describes the variation of the polarization in the sample submitted to an electric field shows the reversible switching of the polarization between 2 opposite states, evidencing the ferroelectric properties of the composite. As stated by M. Josse et al. [24], the room-temperature ferroelectric properties in these ceramics are mainly originating from an off center position of the niobium ions within the octahedral sites. Even after applying the highest electric field that can be reached by our system without causing a breakdown across the ceramic $\left(E_{\max }=26 \mathrm{kV} / \mathrm{cm}\right)$, the saturation polarization was not reached and the maximum measured value of the polarization is $P_{\max } \approx 0.8 \mu \mathrm{C} \mathrm{cm}^{-2}$. The electric coercive field $\left(E_{C}\right)$ is plotted as a function of the maximum electric field applied as shown in the inset of Fig. 2a, which depicts an increase in $\mathrm{E}_{\mathrm{C}}$ while increasing the applied electric field to reach a maximum measured value of $\approx 16.3 \mathrm{kV} \mathrm{cm}^{-1}$. As known for ferroelectric materials, the increase in $E_{C}$ is mainly related to the movement of ferroelectric domain walls, which depend on the applied electric field. The variation of the switching current as a function of 

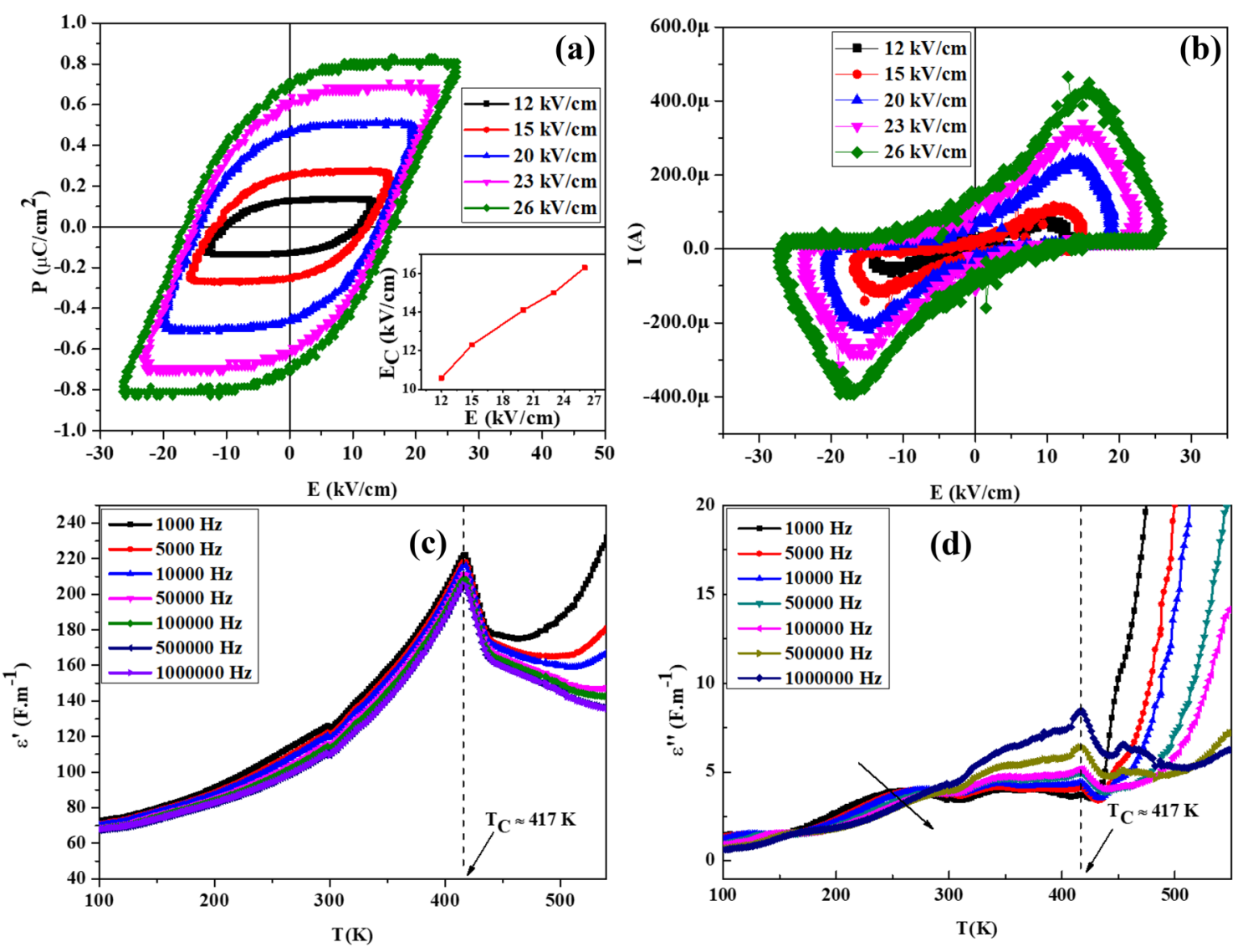

Fig. 2 Room-temperature ferroelectric properties of the synthesized ceramics: $\mathbf{a}$ and $\mathbf{b}$ represent the variation of the polarization and switching current as a function of an applied voltage, respectively, for various electric field amplitudes ranging from $12 \mathrm{kV} / \mathrm{cm}$

to $26 \mathrm{kV} / \mathrm{cm}$. The inset of a shows the variation of the coercive field as a function of the maximum electric field applied. Variation of the dielectric permittivity as a function of temperature and frequency: c real part and $\mathbf{d}$ imaginary part of the dielectric permittivity

the applied electric field is represented in Fig. 2b. The presence of 2 antisymmetric peaks with respect to the current axis gives indication on the coercive field and proves the switching of the ferroelectric polarization. While the variation of the position of the current peaks with the applied field is related to the variation of the coercive field, the increase in its amplitude is related to the increase in the maximum of the measured polarization.

The temperature dependence of the real part of the permittivity $\varepsilon^{\prime}$ at various frequencies ranging from $10^{3}$ to $10^{6} \mathrm{~Hz}$ has been studied, and the results are displayed in Fig. 2c. The real part of the permittivity of the TTB-Sm solid solution possesses a relatively narrow peak at a temperature around $417 \mathrm{~K}$. The position of this peak indicates that the ceramic undergoes a paraelectric-ferroelectric phase transition at a Curie temperature around $T_{C} \approx 417 \mathrm{~K}$. The unchanged position of the peak while increasing the frequency of the measurement is a solid evidence to the ferroelectric nature of the ceramic, confirming the results of the P-E hysteresis loops shown in Fig. 2a. We found that Curie temperature determined here by dielectric

measurements is slightly higher than that determined in [24] $\left(T_{C} \approx 405 \mathrm{~K}\right)$. In the paraelectric region $\left(T>T_{C}\right)$, the real part of the permittivity does not obey the linear behavior expected from the Curie-Weiss law in the paraelectric state, as a change of slope is obvious around $430 \mathrm{~K}$. This can be related to the behavior observed in the $\mathrm{Ba} 2 \mathrm{NbFe}\left(\mathrm{Nb}_{1-\mathrm{x}} \mathrm{Ta}_{\mathrm{x}}\right)_{4} \mathrm{O}_{15}$ solid solution, and to the existence of a metastable ferroelectric phase that appear as a general feature in this TTB series $[26,38]$.

The temperature dependence of the imaginary part of the dielectric permittivity $\left(\varepsilon^{\prime \prime}\right)$ at various frequencies has also been measured and is depicted in Fig. 2d. A peak having a maximum position independent to the frequency at $T=T_{C}$ is obtained, which is clearly related to the paraelectric-ferroelectric phase transition. Moreover, a second dielectric anomaly is observed at lower temperature of around $250 \mathrm{~K}$, suggesting a second phase transition at reduced temperature that can be related to the recurrent ferroelectric trelaxor crossovers observed in this series [24, $26,39]$. The presence of a very wide peak extending over a large frequency range indeed suggests the occurrence 
of a dielectric relaxation at reduced temperature $[40,41]$. Finally, the imaginary part of the permittivity increases drastically at high temperatures $(T>432 \mathrm{~K})$, which reflects the presence of extrinsic contributions related to the establishment of a significant electrical conductivity [42]. This dielectric investigation, however, confirms that the studied ceramics are ferroelectric at room temperature.

In order to investigate the microelectromechanical properties of the studied ceramics and get space-resolved information about their ferroelectric properties at room temperature, piezoelectric force microscopy was used. The out-of-plane and the in-plane piezoelectric responses (mixed signal, i.e., amplitudex $\cos$ (phase)) are shown in Fig. 3a, d, respectively. First, the bright and dark contrasts in Fig. 3a are, respectively, attributed to polar regions where the out-of-plane component of the polarization is oriented upward $(\bigcirc)$ and downward $(\otimes)$ perpendicular to the plane of the image, i.e., the surface of the ceramic sample. Second, the bright and dark contrasts observed in Fig. $3 \mathrm{~b}$ indicate the presence of polar regions where the polarization is lying in the plane of the sample surface and oriented in opposite directions, as depicted by the arrows $(\uparrow \downarrow)$. These contrasts evidence the out-of-plane and the in-plane piezoelectric vibrations, thus the piezoelectric properties of the studied material.

In order to get more information about the local ferroelectric and piezoelectric properties of the studied system at room temperature, we evaluated the variation of the longitudinal $\left(d_{\mathrm{zz}}\right)$ and the transversal $\left(d_{\mathrm{xz}}\right)$ piezoelectric coefficients $[43,44]$ by cycling an external voltage via the
PFM tip fixed above selected locations on the sample surface. The dependence of $d_{\mathrm{zz}}$ and $d_{\mathrm{xz}}$ on the applied bias pulse is described by a hysteresis loop as shown in Figs. $3 c$, $d$, respectively. The well-saturated character of the hysteresis curves proves that the polarization can be reversibly switched between 2 opposite stable states, which clearly confirms the ferroelectric nature of the material. This result suggests that this material could possibly be used for memory devices that require the polarization to switch between two states with an angle of $180^{\circ}$ [45]. The maximum value of piezoelectric coefficients $d_{\mathrm{zz}}$ and $d_{\mathrm{xz}}$ are estimated at $1.3 \mathrm{pm} / \mathrm{V}$ and $1 \mathrm{pm} / \mathrm{V}$, respectively. The difference of coercive voltages in both positive and negative directions is related to the fact that the top electrode (the AFM/PFM tip) has both a very different geometry and a different composition than the continuous bottom electrode [46]. The average coercive voltage is similar in both cases of piezoelectric coefficients, which is estimated at $\approx 4.5 \mathrm{~V}$.

In order to determine the nature and the spatial distribution of the magnetic phase embedded in the ferroelectric matrix, the magnetic properties have been studied at macroscopic and microscopic levels, as shown in Fig. 4. First, the hysteresis loop describing the variation of the macroscopic magnetization as a function of an applied magnetic field was studied at room temperature as depicted in Fig. 4a, evidencing of the presence of a room-temperature ferromagnetic ordering in the studied TTB-Sm/BaFO ceramic composite. The magnetic coercive field $\left(H_{C}\right)$ measured to be around 2200 Oe $(0.22 \mathrm{~T})$ corresponds to the magnetic coercive field of the in situ
Fig. 3 Microscopic electromechanical properties of TTB-Sm ceramics investigated at room temperature: $\mathbf{a}$ and $\mathbf{b}$ show, respectively, the out-of-plane and the in-plane piezoelectric responses detected at the ceramics surface. $c$ and d represent the applied voltage dependence of the out-of-plane and the in-plane piezoelectric coefficients, respectively
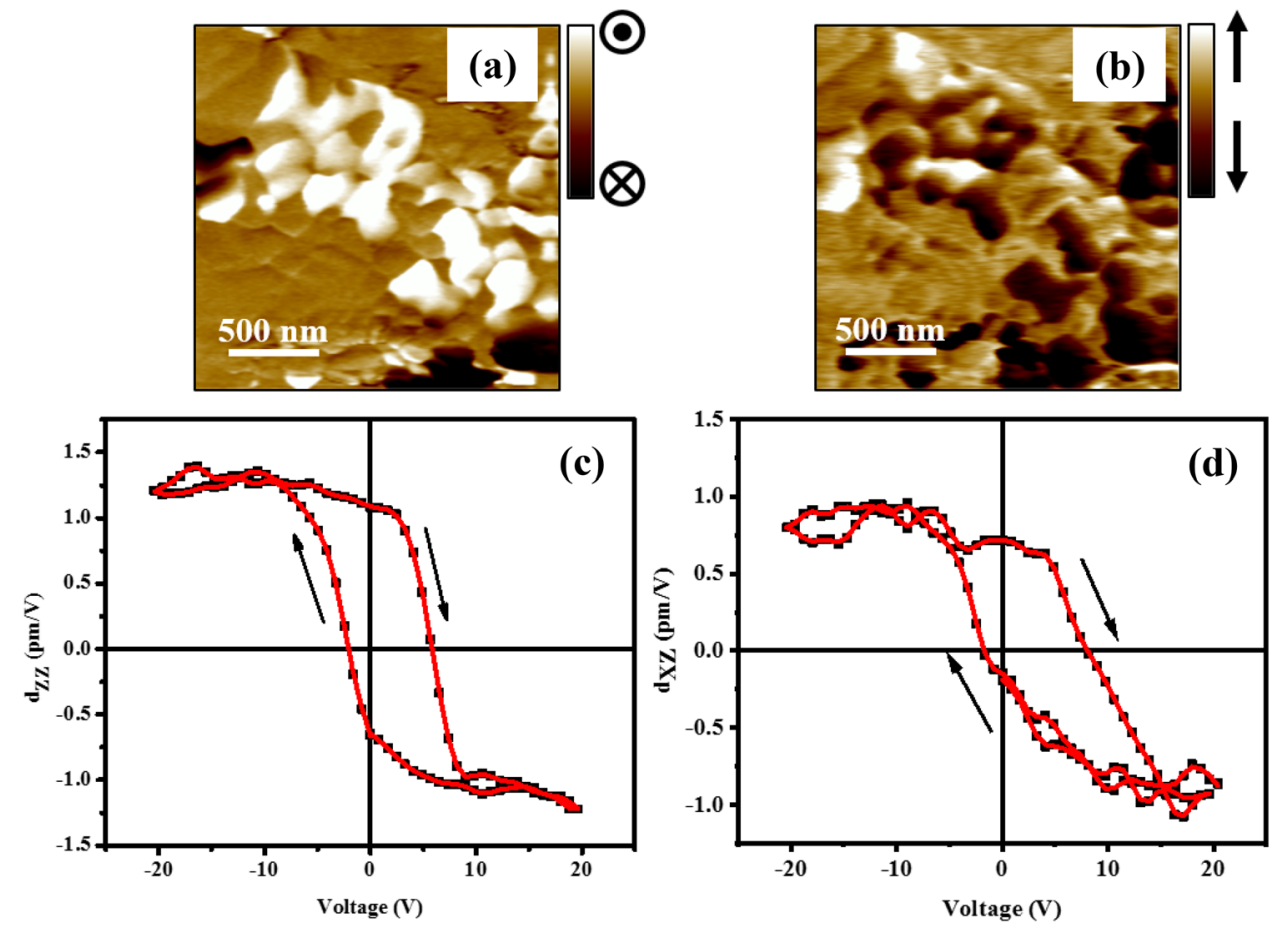

SN Applied Sciences A SPRINGER NATURE journal 

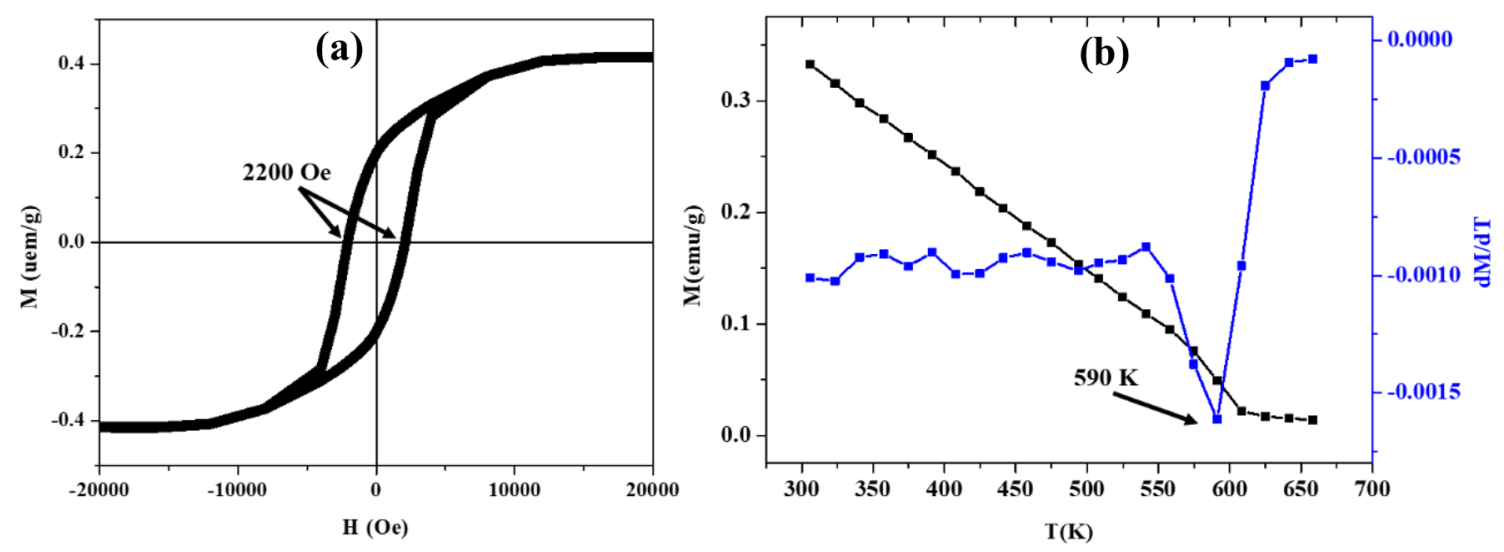

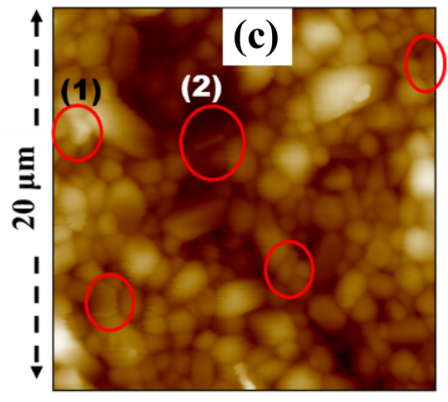

Topography

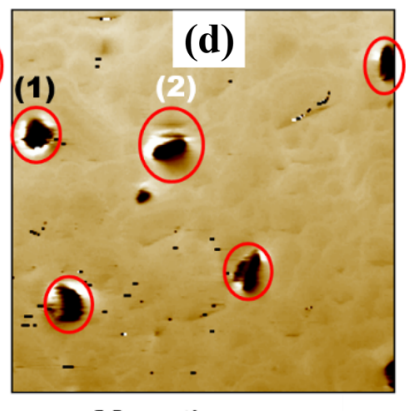

Magnetic response

as a function of temperature. $\mathbf{c}$ and $\mathbf{b}$ Topography and the microscopic magnetic response of the sample surface, respectively, measured at room temperature, showing the magnetic domains distribution at the ceramic surface

To determine the spatial distribution of the magnetic phase at the sample surface, magnetic force microscopy was used. The surface topography and the magnetic response measured at room temperature are shown in Figs. 4 c, d, respectively. The image of the topography (Fig. 4c) shows that the microstructure of the ceramic is formed by a grain structure of lateral dimensions varying from 0.8 to $3 \mu \mathrm{m}$, giving rise to a roughness of about $\mathrm{RMS} \approx 250 \mathrm{~nm}$. Since the material studied in the present research is a polycrystalline ceramics and that the average crystallite size determined by the analysis of the XRD spectra was $35 \mathrm{~nm}$, each grain, composed of several crystallites, is formed on average by $\frac{\text { average grain size }}{\text { crystallite size }}=55$ crystallites. The presence of domains with dark contrast in the image of the magnetic response (Fig. 4d) reveals the presence of small localized magnetic areas with magnetic contrast within a non-magnetic matrix (the ferroelectric matrix TTB-Sm), at locations indicated by the red circles in Figs. $4 \mathrm{c}$ and d. A careful comparison between these 2 images - comparing the circled areas (1) and (2) in Figs. $4 \mathrm{c}, \mathrm{d} d$-indicates the absence of any correlation between the topography and the magnetic response. larger $[47,48]$. 
Each magnetic domain (with a uniform contrast in the MFM image) extends over several grains (having different heights, reflected by the varying contrast in the topographic AFM image). This confirms that the contrasts observed in Fig. $4 \mathrm{c}$ are solely due to the presence of magnetic regions at or near the sample surface. Since there is no obvious morphological difference in the grains located where a magnetic response has been observed and the rest of the grains, this suggests that the presence of the regions with magnetic properties observed is not directly located at the surface, but buried close to the surface, embedded in the ferroelectric matrix. Owing to the fact that hints of the presence of the BaFO phase have been found in the XRD diffractogram and that the magnetic properties measured correspond to those of $\mathrm{BaFO}$, we conclude that the observed contrast is related to the magnetic response of the $\mathrm{BaFO}$ phase. The average size of the magnetic domains (which we associate with the BaFO phase) is around $1.38 \mu \mathrm{m}$, indicating that these magnetic domains are probably formed by several grains of BaFO. This suggests that, like in the case of other TTB-Ln ceramics [24, 28, 29], a magnetic BaFO phase is indeed spontaneously formed dispersed within the TTB-Sm matrix during its thermal treatment of TTB-Sm, and demonstrates that the magnetic properties of these small magnetic regions can be experimentally observed, giving rise to a composite ceramic multiferroic at room temperature.

\section{Conclusion}

Multiferroic composite ceramics TTB-Sm/BaFO were synthesized, and their multifunctional properties were investigated. The ferroelectric and the magnetic phase transition temperatures were determined to be $417 \mathrm{~K}$ and $590 \mathrm{~K}$, respectively. In addition, ferroelectric and ferromagnetic hysteresis loops were measured at room temperature. The microelectromechanical characterization confirmed the presence of ferroelectric properties down to nanoscale and allowed the determination of the piezoelectric coefficients. The formation of the magnetic phase in the form of microdomains was confirmed by studying the magnetic response of the ceramic at the microscopic level. In this study, not only the multiferroic properties of the TTB-Sm/ BaFO composite ceramics have been investigated, but the distribution of the polar and magnetic properties was locally studied as well. Finally, the coexistence of the polar and magnetic domains in the same material suggests the investigation of possible couplings between these domains (magnetoelectric coupling) as a prospective for this study.
Funding This research was funded by the Natural Sciences and Engineering Research Council of Canada (NSERC) Individual Discovery Grant \# RGPIN 261662.

\section{Compliance with ethical standard}

Conflict of interest The authors declare that they have no competing interests.

\section{References}

1. Spaldin,. Cheong, . Ramesh, NASR (2010) Multiferroics : past, present, and future feature. Phys Today 63(9):38-43. https:// doi.org/10.1063/1.3502547

2. Lu C, Hu W, Tian Y, Wu T (2015) Multiferroic oxide thin films and heterostructures. Appl Phys Rev 2:021304. https://doi. org/10.1063/1.4921545

3. Ramesh R, Spaldin NA (2007) Multiferroics: progress and prospects in thin films. Nat Mater 6:21-29. https://doi.org/10.1038/ nmat 1805

4. Eerenstein W, Mathur ND, Scott JF (2006) Multiferroic and magnetoelectric materials. Nature 442759:759-765. https://doi. org/10.1038/nature05023

5. Nan CW, Bichurin MI, Dong S, Viehland D, Srinivasan G (2008) Multiferroic magnetoelectric composites: historical perspective, status, and future directions. J Appl Phys 103:1-35. https://doi. org/10.1063/1.2836410

6. Turchenko VA, Trukhanov SV, Balagurov AM, Kostishyn VG, Trukhanov AV, Panina LV, Trukhanova EL (2018) Features of crystal structure and dual ferroic properties of BaFe12-xMexO19 (Me=In3+ and Ga3+; $x=0.1-1.2$ ). J. Magn Magn Mater. 464:139147. https://doi.org/10.1016/j.jmmm.2018.05.036

7. Salem MM, Panina LV, Trukhanova EL, Darwish MA, Morchenko AT, Zubar TI, Trukhanov SV, Trukhanov AV (2019) Structural electric and magnetic properties of (BaFe11 9AI0 1019)1- $x$ - (BaTiO3) x composites. Compos Part B Eng 174(1):107-154

8. Turchenko V, Trukhanov A, Trukhanov S, Balasoiu M, Lupu N (2019) Correlation of crystalline and magnetic structures of barium ferrites with dual ferroic properties. J Magn Magn Mater 477:9-16. https://doi.org/10.1016/j.jmmm.2018.12.101

9. Spaldin NA, Ramesh R (2019) Advances in magnetoelectric multiferroics. Nat Mater 18:203-212. https://doi.org/10.1038/s4156 3-018-0275-2

10. Baek SH, Jang HW, Folkman CM, Li YL, Winchester B, Zhang JX, He Q, Chu YH, Nelson CT, Rzchowski MS, Pan XQ, Ramesh R, Chen $L Q$, Eom CB (2010) Ferroelastic switching for nanoscale non-volatile magnetoelectric devices. Nat Mater 9:309-314. https://doi. org/10.1038/nmat2703

11. Shen J, Cong J, Shang D, Chai Y, Shen S, Zhai K, Sun Y (2016) A multilevel nonvolatile magnetoelectric memory. Sci Rep 6(34473):1-6. https://doi.org/10.1038/srep34473

12. Roy A, Gupta R, Garg A (2012) Multiferroic memories. Adv Condens Matter Phys 2012:1-12. https://doi. org/10.1155/2012/926290

13. Scott JF (2007) Multiferroic memories. Nat Mater 6:256-257

14. Nechache R, Carignan L-P, Gunawan L, Harnagea C, Botton GA, Ménard D, Pignolet A (2011) Epitaxial thin films of multiferroic Bi2FeCrO6 with B-site cationic order. J Mater Res 22:2102-2110. https://doi.org/10.1557/jmr.2007.0273

15. Sanchez DA, Ortega N, Kumar A, Sreenivasulu G, Katiyar RS, Scott JF, Evans DM, Arredondo-Arechavala M, Schilling A, Gregg JM (2013) Room-temperature single phase multiferroic 
magnetoelectrics: $\mathrm{Pb}(\mathrm{Fe}, \mathrm{M}) \times(\mathrm{Zr}, \mathrm{Ti})(1-\mathrm{x}) \mathrm{O} 3[\mathrm{M}=\mathrm{Ta}, \mathrm{Nb}]$. J Appl Phys 113:1-7. https://doi.org/10.1063/1.4790317

16. Zhao HJ, Ren W, Yang Y, Iniguez J, Chen XM, Bellaiche L (2014) Near room-temperature multiferroic materials with tunable ferromagnetic and electrical properties. Nat Commun. 5(4021):1-7. https://doi.org/10.1038/ncomms5021

17. Scott JF (2013) Room-temperature multiferroic magnetoelectrics. NPG Asia Mater 5:1-11. https://doi.org/10.1038/ am. 2013.58

18. Jayachandran KP, Guedes JM, Rodrigues HC (2018) Solutions for maximum coupling in multiferroic magnetoelectric composites by material design. Sci Rep 8(4866):1-9. https://doi.org/10.1038/ s41598-018-22964-9

19. Ma J, Hu J, Li Z, Nan CW (2011) Recent progress in multiferroic magnetoelectric composites: From bulk to thin films. Adv Mater 23:1062-1087. https://doi.org/10.1002/adma.201003636

20. Ai D, Xu J, Huang C, Zhou W, Zhao L, Sun J, Wang Q (2017) Synthesis and magnetoelectric properties of multiferroic composites of lead lanthanum zirconate titanate and mesoporous cobalt ferrite. Scr Mater 136:29-32. https://doi.org/10.1016/j. scriptamat.2017.04.003

21. Gao R, Qin X, Zhang Q, Xu Z, Wang Z, Fu C, Chen G, Deng X, Cai W (2019) A comparative study of the dielectric, ferroelectric and anomalous magnetic properties of $\mathrm{Mn} 0.5 \mathrm{Mg} 0.5 \mathrm{Fe} 2 \mathrm{O} 4 /$ Ba0.8Sr0.2Ti0.9Zr0.103 composite ceramics. Mater Chem Phys 232:428-437. https://doi.org/10.1016/j.matchemphy s.2019.05.016

22. Laguta VV, Elissalde C, Maglione M, Artemenko AM, Chlan V, Stepankova H, Zagorodniy Y (2015) Crystal structure transformations induced by surface stresses in $\mathrm{BaTiO} 3$ and $\mathrm{BaTiO} 3 @ S i O 2$ nanoparticles and ceramics. Phase Transitions 88:761-775. https ://doi.org/10.1080/01411594.2014.996852

23. Chung UC, Michau D, Elissalde C, Li S, Klein A, Maglione M (2012) Evidence of diffusion at BaTiO3/silicon interfaces. Thin Solid Films 520:1997-2000. https://doi.org/10.1016/j.tsf.2011.09.055

24. Josse M, Bidault O, Roulland F, Castel E, Simon A, Michau D, Von der Muhll R, Nguyen O, Maglione M (2009) The Ba2LnFeNb4O15 tetragonal tungsten bronze: towards RT composite multiferroics. Solid State Sci 11:1118-1123. https://doi.org/10.1016/j.solid statesciences.2009.02.015

25. Aamlid SS, Selbach SM, Grande T (2019) The Effect of Cation disorder on ferroelectric properties of SrxBa1-xNb2O6 tungsten bronzes. Materials (Basel) 12:1-11. https://doi.org/10.3390/ ma12071156

26. Josse $M$, Heijboer $P$, Albino $M$, Molinari F, Porcher F, Decourt $R$, Michau D, Lebraud E, Veber P, Velazquez M, Maglione M (2014) Original crystal-chemical behaviors in ( $\mathrm{Ba}, \mathrm{Sr}) 2 \mathrm{Ln}(\mathrm{Fe}, \mathrm{Nb}$, Ta)5O15 tetragonal tungsten bronze: anion-driven properties evidenced by cationic substitutions. Cryst Growth Des 14:54285435. https://doi.org/10.1021/cg5006049

27. Yang Z, Wei L, Yang B, Yang P, Li J (2020) Effects of A-site cations on the electrical behaviors in (Sr1-xCax) $21 \mathrm{Na} 0$ 8Nb5015 tungsten bronze ferroelectrics. Mater Chem Phys 243:122006. https ://doi.org/10.1016/j.matchemphys.2019.122006

28. Roulland F, Josse M, Castel E, Maglione M (2009) Influence of ceramic process and Eu content on the composite multiferroic properties of the Ba6-2xLn2xFe1+xNb9-xO30 TTB system. Solid State Sci 11:1709-1716. https://doi.org/10.1016/j.solidstatescien ces.2009.05.031

29. Castel E, Josse M, Roulland F, Michau D, Raison L, Maglione M (2009) In-situ formation of barium ferrite in iron-doped tetragonal tungsten bronze: elaboration of room temperature multiferroic composites. J Magn Magn Mater 321:1773-1777. https:// doi.org/10.1016/j.jmmm.2009.02.010

30. Li C, Huang YH, Hong JS, Liu XQ, Li J, Wu YJ (2019) Magnetoelectric effect in Sm-substituted tungsten bronze structure
Ba4(SmxLa1-x)2Fe2Nb8O30 ceramics. J Alloys Compd 786:126133. https://doi.org/10.1016/j.jallcom.2019.01.242

31. Scherrer P (1918) Bestimmung der Größe und der inneren Struktur von Kolloidteilchen mittels Röntgenstrahlen. Göttinger Nachrichten Math Phys 2:98-100

32. Hajlaoui T, Harnagea C, Michau D, Josse M, Pignolet A (2017) Highly oriented multiferroic Ba 2 NdFeNb 4 O 15 -based composite thin films with tetragonal tungsten bronze structure on silicon substrates. J Alloys Compd 711:480-487. https://doi. org/10.1016/j.jallcom.2017.04.051

33. Bovtun V, Kamba S, Veljko S, Nuzhnyy D, Knížek K, Savinov M, Petzelt J (2007) Relaxor-like behavior of lead-free Sr2LaTi2Nb 3015 ceramics with tetragonal tungsten bronze structure. J Appl Phys 101:1-7. https://doi.org/10.1063/1.2713094

34. M. Josse, Des fluorures aux ferroïques, l'empire de la cristallochimie, Université de Bordeaux, 2012. https://tel.archivesouvertes.fr/tel-00842197/

35. Z. CHEN, F. YANG, Barium hexa-ferrite technology for MAMIR and advanced magnetic recording applications, 2017, 14/799,313.

36. Sharrock MP, Carlson LW (1995) Application of barium ferrite particles in advanced recording media. IEEE Trans Magn 31:2871-2876. https://doi.org/10.1109/20.490177

37. Ozah S, Bhattacharyya NS (2013) Nanosized barium hexaferrite in novolac phenolic resin as microwave absorber for X-band application. J Magn Magn Mater 342:92-99. https://doi. org/10.1016/j.jmmm.2013.04.050

38. Albino $M$, Heijboer $P$, Porcher F, Decourt R, Veber P, Maglione $M$, Josse M (2018) Metastable ferroelectric phase and crossover in the Ba2NdFeNb4-xTaxO15 TTB solid solution. J Mater Chem C 6:1521-1534. https://doi.org/10.1039/c7tc04742h

39. Castel E, Josse M, Michau D, Maglione M (2009) Flexible relaxor materials: Ba2PrxNd1-xFeNb4O15 tetragonal tungsten bronze solid solution. J Phys Condens Matter 21:452201. https://doi. org/10.1088/0953-8984/21/45/452201

40. A Peláiz-Barranco, F Calderón-Piñar, O García-Zaldívar, Y González-Abreu (2016), Relaxor Behaviour in Ferroelectric Ceramics Chapter, In: Adv Ferroelectr, pp. 85-107.InTech, Rijeka.https://doi.org/10.5772/47751.hancement

41. Cross LE (1994) Ferroelectrics Relaxor ferroelectrics : an overview. Ferroelectrics 151:305-320

42. Zhang TF, Tang XG, Liu QX, Lu SG, Jiang YP, Huang XX, Zhou QF (2014) Oxygen-vacancy-related relaxation and conduction behavior in (Pb1-xBax)(Zr0 95Ti0 05)O3 ceramics. AIP Adv 4(107141):1-11. https://doi.org/10.1063/1.4900610

43. Harnagea C, Cojocaru CV, Pignolet A (2005) Local ferroelectric switching properties in $\mathrm{BiFeO} 3$ microstructures and their piezomagnetic response. MRS Proc 902:1-6. https://doi.org/10.1557/ PROC-0902-T10-57

44. Harnagea C, Cojocaru CV, Nechache R, Gautreau O, Rosei F, Pignolet A (2008) Towards ferroelectric and multiferroic nanostructures and their characterisation. Int J Nanotechnol 5:930-962

45. Palneedi H, Annapureddy V, Priya S, Ryu J (2016) Status and perspectives of multiferroic magnetoelectric composite materials and applications. Actuators 5:1-31. https://doi.org/10.3390/ act5010009

46. Hajlaoui T, Corbellini L, Harnagea C, Josse M, Pignolet A (2017) Enhanced ferroelectric properties in multiferroic epitaxial Ba2EuFeNb4O15 thin films grown by pulsed laser deposition. Mater Res Bull 87:186-192. https://doi.org/10.1016/j.materresbu II.2016.11.033

47. Trukhanov AV, Trukhanov SV, Kostishin VG, Panina LV, Salem MM, Kazakevich IS, Turchenko VA, Kochervinskii VV, Krivchenya DA (2017) Multiferroic properties and structural features of M-type Al-substituted barium hexaferrites. Phys Solid State 59:737-745. https://doi.org/10.1134/S1063783417040308 
48. Trukhanov AV, Trukhanov SV, Panina LV, Kostishyn VG, Chitanov DN, Kazakevich IS, Trukhanov AV, Turchenko VA, Salem MM (2017) Strong corelation between magnetic and electrical subsystems in diamagnetically substituted hexaferrites ceramics. Ceram Int 43:5635-5641. https://doi.org/10.1016/j.ceram int.2017.01.096
Publisher's Note Springer Nature remains neutral with regard to jurisdictional claims in published maps and institutional affiliations. 